\title{
Ultrasonographic imaging of the reproductive organs of the female bottlenose dolphin, Tursiops truncatus aduncas
}

\author{
F. M. Brook \\ Department of Optometry and Radiography, The Hong Kong Polytechnic University, \\ Kowloon, Hong Kong
}

Routine ultrasonographic examination of the reproductive tract was performed for periods of up to $\mathbf{1 0}$ years in ten female bottlenose dolphins (Tursiops truncatus aduncas) in Hong Kong. The ovaries could be reliably and repeatedly identified, lying close to the body surface, in the angle formed by the rectus abdominus and hypaxialis lumborum muscles, and were most easily located by scanning in the transverse plane from the proximal end of the genital slit towards the head. The ovaries are ovoid, with a relatively hypoechoic cortex around a central echogenic mesovarium. The echogenicity of the ovarian parenchyma appeared to increase with increasing age. This may be the result of age-related changes, such as increased fat deposition or fibrosis, or of ovarian 'scars' from multiple ovulations.
Small antral follicles, developing follicles and corpora lutea can be identified within the ovarian cortex. Owing to its shape and lack of a definitive border, plus the close relationship to the intestines, the contents of which may obstruct the ultrasound beam, the non-pregnant uterus was not so easily visualized. The endometrium was poorly differentiated and difficult to see. To date, examination of the uterus using ultrasonography has provided little information about endometrial changes during the ovarian cycle in this group of dolphins. Real-time diagnostic ultrasonography provides a means to image the morphology of the reproductive organs in live female dolphins directly and provides a valuable means of assessing reproductive events in this species.

\section{Introduction}

Ultrasonography is a sophisticated diagnostic imaging method, with a recognized and valued role in assessment of ovarian morphology, ovulation physiology and management of pregnancy in many species (DeCherney et al., 1982).

The application of real-time ultrasonography in the study of the anatomy and physiology of reproductive organs in exotic, wild or endangered species is increasing. Ultrasonographic imaging of the female reproductive tract has been reported in several large, non-domestic species, including the elephant, rhinoceros, giraffe, banteng and gaur (Adams et al., 1991) and is an increasingly necessary research tool for examining reproductive biology in exotic species.

Bottlenose dolphins (Tursiops truncatus aduncas) have been maintained in captivity for more than a century; however, knowledge of their reproductive physiology remains relatively rudimentary. Ultrasonography has been used to diagnose and monitor pregnancy in a few animals (Stone et al., 1984, 1999; Williamson et al., 1990; Taverne, 1991) but little effort has been made to apply the many advantages of the technique to the study of ovarian dynamics in this species.

Email: orfiona@polyu.edu.hk
Interest in controlled breeding of cetaceans has increased, particularly with regard to genetic management (Robeck et al., 1994) and direct visualization of ovarian events aids breeding management. Robeck et al. (1995) used ultrasonography to monitor ovarian changes in two bottlenose dolphins after ovulation induction protocols and found it was possible to 'serially locate and evaluate' superovulated ovaries; however, these authors did not describe the appearance of normal ovaries. The purpose of the present paper is to describe a simple scanning protocol that enables repeatable visualization of the ovaries of bottlenose dolphins and to describe the ultrasonographic appearance of the normal reproductive organs.

\section{Materials and Methods}

\section{Animals and management}

Ultrasonographic imaging was performed in ten female Tursiops truncatus aduncas held at Ocean Park, Hong Kong: three Taiwanese T. t. aduncas and seven smaller $T$. t. aduncas from Indonesia. Body lengths, weights and estimated ages of all animals are provided (Table 1). All female dolphins were usually housed at the same outdoor site, in which there are five interconnecting tanks; animals were generally allowed free access between these. Diets consisted of different proportions of capelin, sardine, herring and squid, with vitamin and mineral supplements. 
Table 1. Estimated age, known reproductive status, body length, weight and length of left ovary in ten female bottlenose dolphins (Tursiops truncatus aduncas) at Ocean Park, Hong Kong, at the end of 1998

\begin{tabular}{|c|c|c|c|c|c|}
\hline Origin & $\begin{array}{c}\text { Estimated age } \\
\text { (years) }\end{array}$ & $\begin{array}{l}\text { Reproductive } \\
\text { status }\end{array}$ & $\begin{array}{l}\text { Body weight } \\
(\mathrm{kg})\end{array}$ & $\begin{array}{l}\text { Body length } \\
(\mathrm{cm})\end{array}$ & $\begin{array}{l}\text { Mean length of left } \\
\text { ovary without follicle } \\
\text { or CL }(\mathrm{cm}) \text { in non- } \\
\text { pregnant, non- } \\
\text { lactating females }\end{array}$ \\
\hline Taiwan & $>30$ & Mature & 155 & 246 & 6.4 \\
\hline Taiwan & $>25$ & Mature & 170 & 244 & 6.2 \\
\hline Indonesia & $>20$ & Mature & 108 & 216 & 4.8 \\
\hline Indonesia & $>20$ & Mature & 96 & 208 & 5.0 \\
\hline Taiwan & $>15$ & Mature & 161 & 234 & 6.0 \\
\hline Indonesia & $>15$ & Mature & 121 & 220 & 5.2 \\
\hline Indonesia & $>15$ & Mature & 108 & 215 & 4.9 \\
\hline Indonesia & $>10$ & Mature & 102 & 218 & 5.1 \\
\hline Indonesia & $>8$ & Mature & 100 & 218 & 5.0 \\
\hline Indonesia & $>7$ & Mature & 100 & 210 & 4.8 \\
\hline
\end{tabular}

$\mathrm{CL}$ : corpus luteum.

\section{Equipment}

Examinations were performed with either an Aloka SSD 630 or 1700 ultrasound unit (Aloka Co. Ltd, Mitakashi, Tokyo) using a $3.5 \mathrm{MHz}$ linear or a $5 \mathrm{MHz}$ curvilinear transducer. Hard copy images were recorded using a thermal printer or by direct digital capture. Because of the nature of the dolphin's skin, there is no air layer between the surface and the transducer so no coupling gel was required.

\section{Behavioural training for ultrasonographic examination of female dolphins}

All female dolphins in this facility were taught to cooperate for ultrasound examination, which made routine and repeatable examinations possible. Initially, the dolphins were taught to approach the poolside and to turn onto either side to present the flank for examination. The trainer supported the tail, which helped stabilize the animal in the water. The ultrasound unit was placed at the poolside during training, so that the animals became used to its presence. After training for poolside approach and presentation, the transducer was introduced and the dolphins were allowed to investigate it. Dolphins appeared to be sensitive to the ultrasonic beam and, at first, animals could be startled and would dart away. With time and positive reinforcement, all dolphins became accustomed to the beam and would usually accept ultrasonographic examination.

\section{Protocol for ultrasonographic examination of the ovaries}

The transducer was placed in the transverse plane midway along the genital slit and moved dorsally along the line where the junction between the triangular, lateral border of the rectus abdominus muscle and the rounded visceral surface of the hypaxialis lumborum muscle can be seen (Fig. 1). The ovaries usually lie between these, against the wall of the abdominal cavity, at a variable distance from the genital slit. There is a palpable depression in the flank where these muscles meet, which can also provide a guide for placement of the transducer (Fig. 2). The transducer was moved cranially until the transverse axis of the ovary was identified. The transducer could then be rotated by $90^{\circ}$ to visualize the long axis of the ovary. It was sometimes necessary to angle the transducer slightly dorsally if the ovary lay partially under the hypaxialis lumborum. The procedure was repeated for the contralateral ovary.

\section{Protocol for ultrasonographic examination of the uterus}

The transducer was placed in the longitudinal plane, just dorsal to the genital slit and moved dorsally until the cervix could be visualized lying just dorsal to the urinary bladder. The transducer was then moved cranially, with short dorso-ventral sweeps to examine the uterine body and ipsilateral horn. The procedure was repeated on the other side.

\section{Results}

\section{Ultrasonographic appearance of the uterus}

The uterine cervix could be clearly visualized by ultrasonography, angled dorsally, immediately behind and usually to one side of the urinary bladder. Sometimes, there was sufficient mucus present in the cervical canal to delineate the internal architecture and allow ultrasonographic visualization of the pseudocervix and spermothecal recess (Fig. 3). The amount of mucus present did not appear to be related to ultrasonographic evidence of 


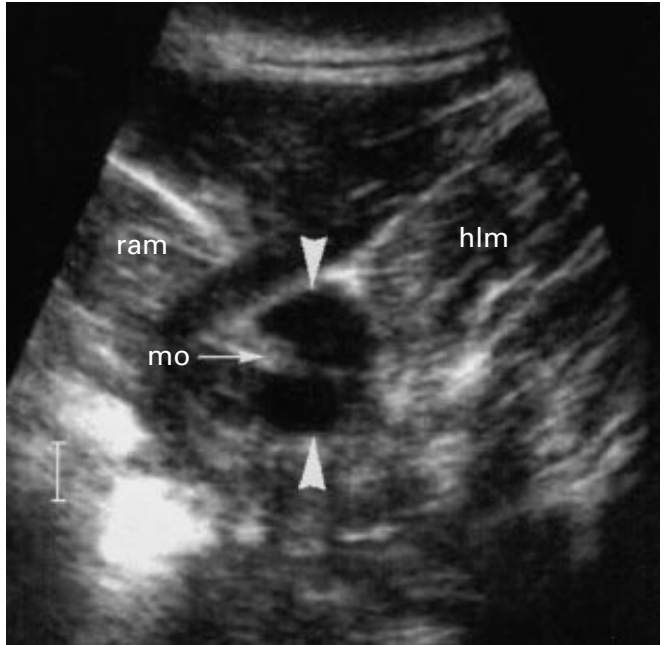

Fig. 1. Transverse ultrasonograms showing a cross-section of the left ovary in the bottlenose dolphin (Tursiops truncatus aduncas). The dolphin is in right lateral recumbence and therefore the ventral abdomen is to the left of the image and the dorsal fin is to the right. The ovary (between the arrowheads) is a rounded, relatively echolucent structure lying between the rectus abdominus (ram) and hypaxial $(\mathrm{hlm})$ muscles. The insertion of the mesovarium (mo) appears as an echogenic area at the centre of the ovary. Scale bar represents approximately $1.0 \mathrm{~cm}$.

ovarian activity nor to serum reproductive hormone concentrations.

The bicornuate uterus is large and is not usually visualized in its entirety, because of the surrounding gut. The myometrium is relatively thin and not well demarcated. The uterine horns curve backwards along their length, to lie in close apposition to the ovaries (Fig. 4). The normal uterus has been shown to be soft and very malleable and presents a rather amorphous, soft tissue mass on ultrasonography. The parenchymal echopattern is homogeneous and of low-to-mid-intensity echogenicity (Fig. 5). In the population studied it was not possible routinely to distinguish the endometrium (Fig. 6) in sufficient detail to be able to characterize the ultrasonographic appearance, or to detect any consistent change during periods of ovarian activity.

\section{Ultrasonographic appearance of the ovaries}

It was possible routinely to identify and assess the ovaries of all females studied, although this could be more difficult in old or fat females, or when ovaries were inactive due to lactation or prolonged periods of anoestrus. The ovaries in Tursiops are relatively superficial, tucked high in the dorso-lateral aspect of the abdomen, to lie in the angle formed by the hypaxialis lumborum and rectus abdominus. The ovarian cortex is relatively echolucent, and is well delineated by an echogenic border that probably represents the tunica albuginea. The ovaries of sexually mature females are ovoid in outline, rounded in cross-section and

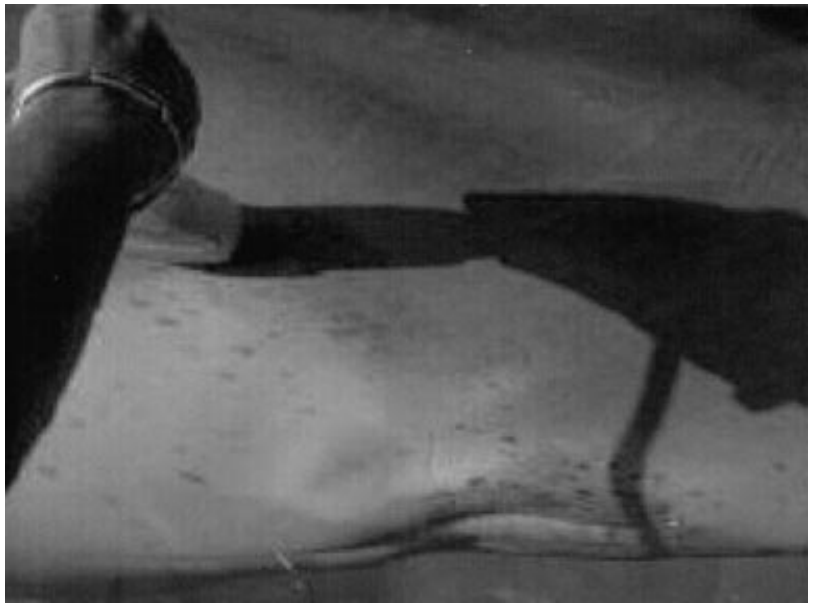

Fig. 2. Photograph indicating the positioning of the transducer for a longitudinal scan of the left ovary in relation to the genital slit in the bottlenose dolphin (Tursiops truncatus aduncas).

of varying echogenicity. The cortex in the older females (Fig. 7) tended to be more echogenic and the outline more irregular than it was in younger animals (Fig. 8). In early 1990, the ovaries in one young female (estimated age $>6$ years) were more angular, and markedly echolucent (Fig. 8). At this time, the serum progesterone concentration recorded for this dolphin was $<0.2 \mathrm{ng} \mathrm{ml}^{-1}$ and she was considered to be juvenile. This ultrasonographic appearance may indicate sexually immature ovaries in $T$. $t$. aduncas. The shape and appearance of the ovaries in the same animal 3 years later (Fig. 9), after five documented ovulations (the first in May 1991) and one pregnancy, was similar to those of other females.

Two echogenic lines represented the reflections of the mesovarium (Figs 7-9) and this characteristic 'tram line' appearance of the long axis of the ovarian hilum was a useful marker for identifying the ovary. Small, echolucent areas were sometimes seen within the hilum of some ovaries and probably represented blood or lymphatic vessels in the mesovarium. At times, free fluid was seen within the hilum. The presence of this fluid was variable and did not coincide with periods of ovarian activity, but probably represented normal peritoneal fluid.

Ovarian lengths in all sexually mature females showed some fluctuation over time; ovaries were slightly longer during periods of ovarian activity, and decreased in size during prolonged periods of anoestrus. The range of ovarian lengths recorded in the present study in non-pregnant, nonlactating females up to the end of 1999 varied from 4.4 to $6.4 \mathrm{~cm}$ (Table 1) and there was a significant correlation $(P=0.038)$ between mean ovarian length and body length (Fig. 10). In all animals, the left ovary was the same size, or slightly longer than the right ovary; cross-sectional diameters were not routinely measured but appeared similar. 


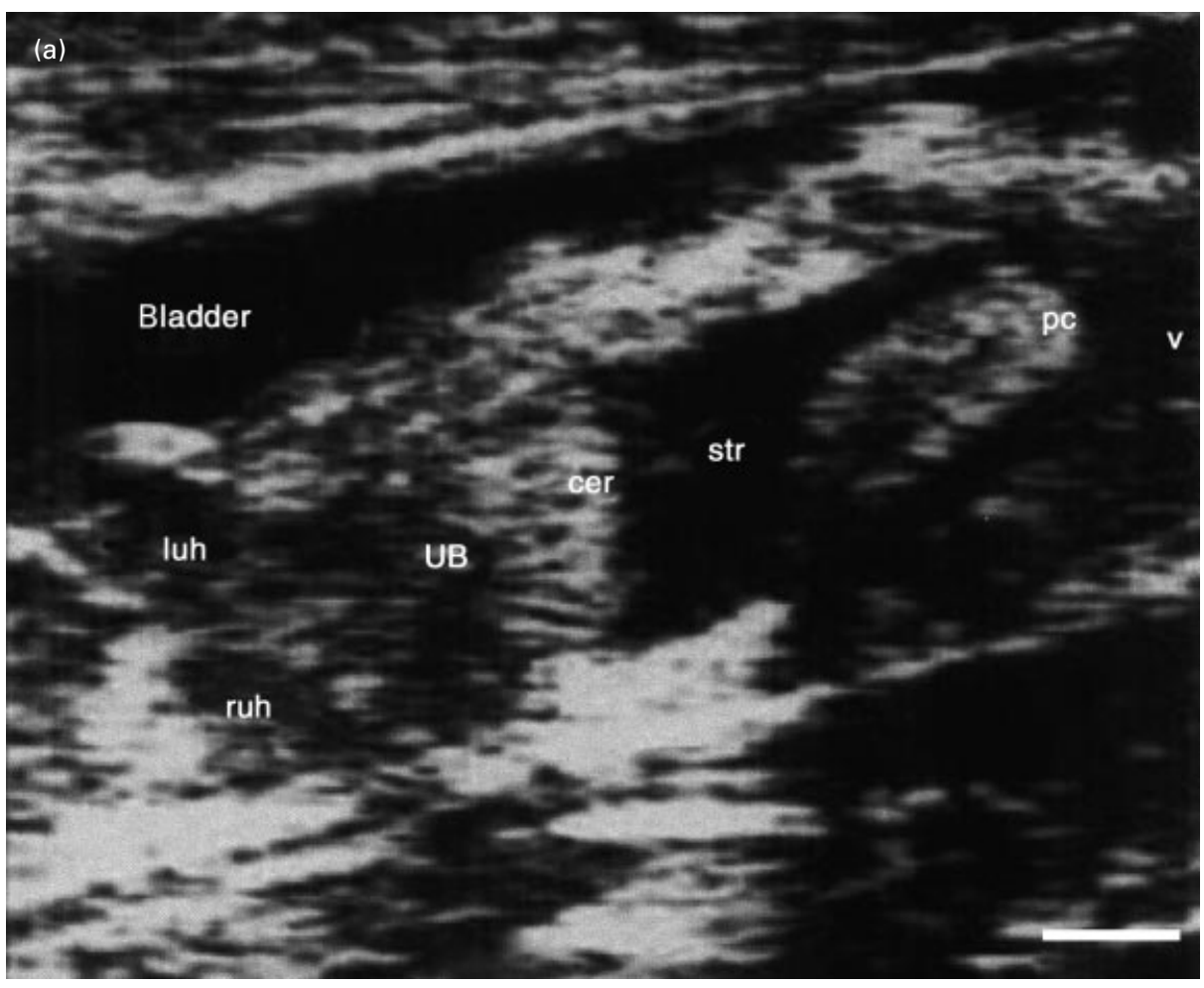

(b)

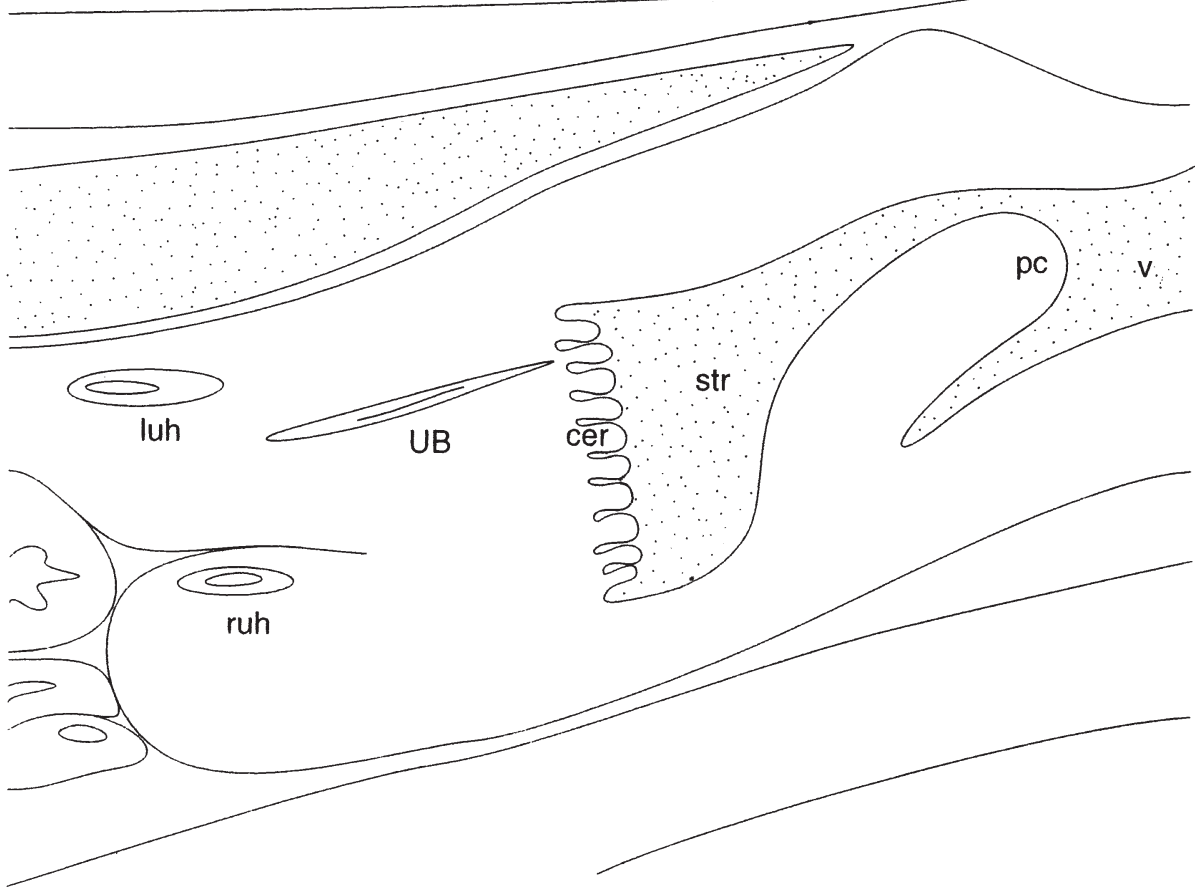

Fig. 3. (a) Ultrasonogram of a longitudinal section of the uterus of the bottlenose dolphin (Tursiops truncatus aduncas) seen behind the partially filled urinary bladder. (b) Line diagram of (a). cer: true cervix; luh: left uterine horn; pc: pseudocervix; ruh: right uterine horn; str: spermothecal recess; UB: uterine body; v: vagina. Scale bar represents $1 \mathrm{~cm}$. 


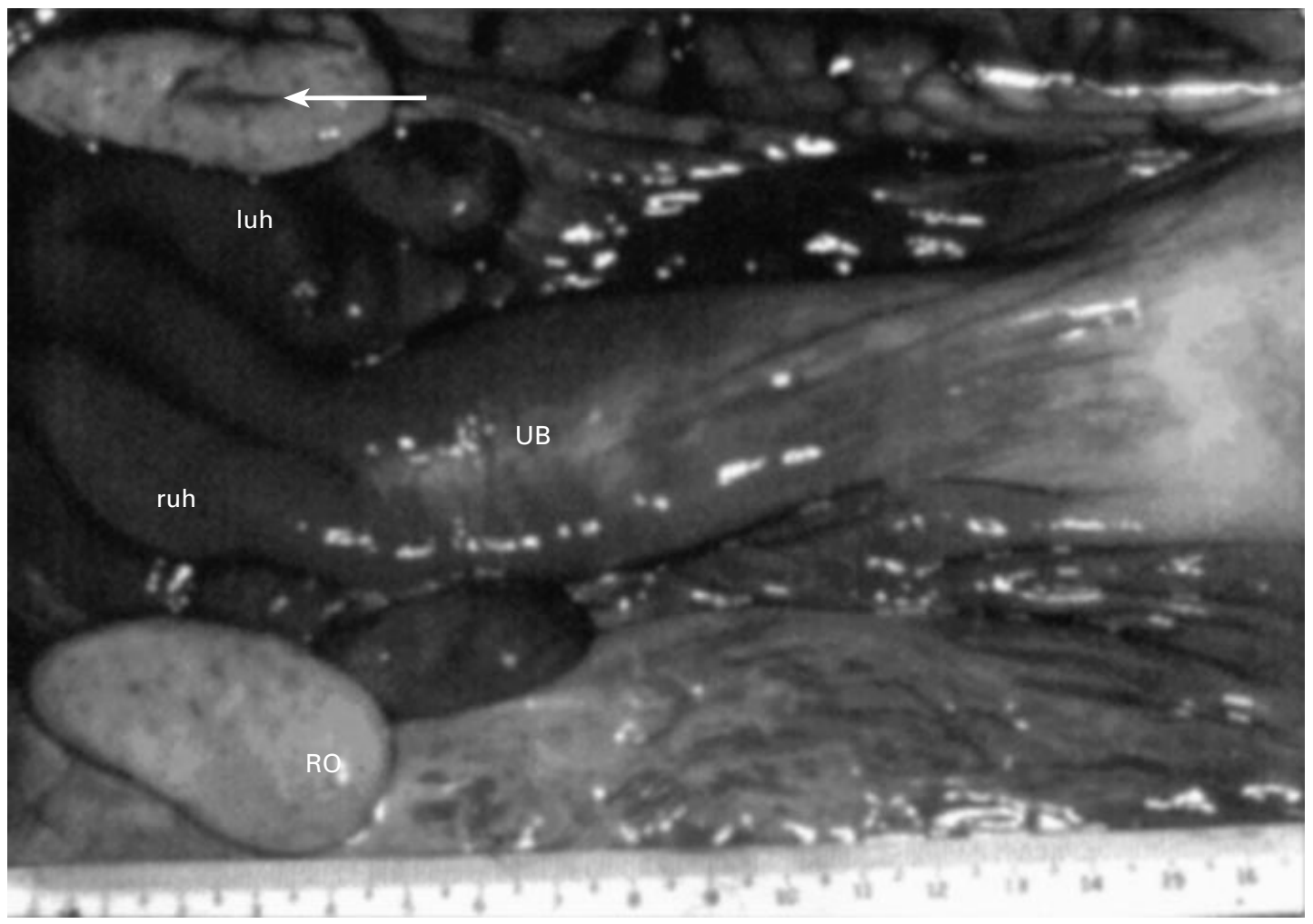

Fig. 4. The uterus and ovaries of the bottlenose dolphin (Tursiops truncatus aduncas) in situ. Arrow indicates the left ovarian hilum, where the mesovarium inserts. luh: left uterine horn; RO: right ovary; ruh: right uterine horn; UB: uterine body. Scale shown at bottom of figure is in $1.0 \mathrm{~cm}$ increments.

Small cystic structures of $2-3 \mathrm{~mm}$ in diameter were often visualized in the ovarian cortex (Fig. 9) and probably represented antral follicles. Antral follicles of up to $4 \mathrm{~mm}$ in diameter were occasionally seen during anoestrus. More than 90 spontaneous ovulations have been documented in this population to date. Developing follicles are always prominent and easily identified using ultrasonography, even in old and fat dolphins, when it is sometimes difficult to distinguish the ovary clearly. A developing follicle presents the classic, rounded, cystic appearance seen in most mammals (Figs 5 and 11a). The diameter of follicles just before ovulation varied among individuals, ranging in the present population from 1.6 to $2.3 \mathrm{~cm}$, but was consistent within individuals.

The site of ovulation (Fig. 11b) or the developing corpus luteum (Fig. 11c,d) could be seen using ultrasonography. The usual appearance of a corpus luteum was a rounded, solid mass at the site of the ruptured follicle, which might appear hypoechoic, isoechoic, or slightly more echogenic than the ovarian parenchyma. Hypoechoic corpora lutea could be distinguished from follicles by their larger size, thicker or irregular walls and lack of distal acoustic enhancement. Occasionally, and particularly if associated with pregnancy, the corpus luteum would cavitate. In the present population, 13 pregnancies have been observed and monitored by ultrasonography. Corpora lutea in early pregnancy (up to week 14 of gestation) tended to be larger (24-39 mm; $n=13$ ) than those of non-fertile cycles $(18-32 \mathrm{~mm} ; n=69)$ and appeared hypoechoic, organized and more regular in outline. In later pregnancy, the corpus luteum decreases slightly in size, but the central cavitation appears to persist (Fig. 12). Ultrasonographic identification of a large, hypoechoic, organized, or cavitated corpus luteum may be a useful diagnostic indicator of early pregnancy in dolphins.

\section{Discussion}

Unlike earlier reports of ultrasonographic examination of captive Tursiops (Stone, 1990; Williamson et al., 1990; Taverne, 1991), the dolphins in the present study generally co-operated well in poolside scanning sessions after sufficient training, and all ovarian assessments were conducted with the dolphins remaining in the water. Female dolphins accustomed to ultrasound examination remained at the poolside for periods of several minutes, which was usually more than adequate for ovarian assessment. If a longer scanning time was needed, the 


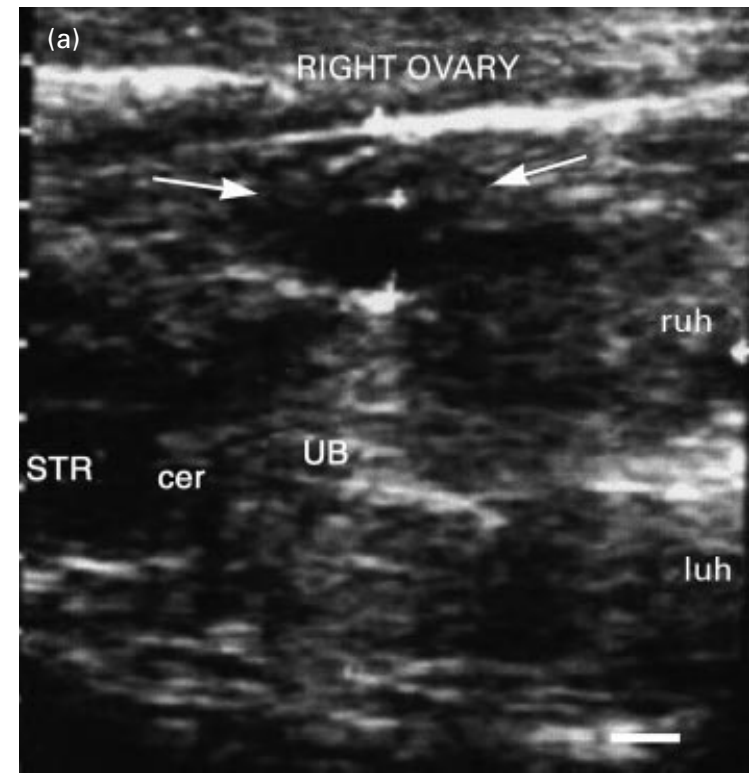

(b)

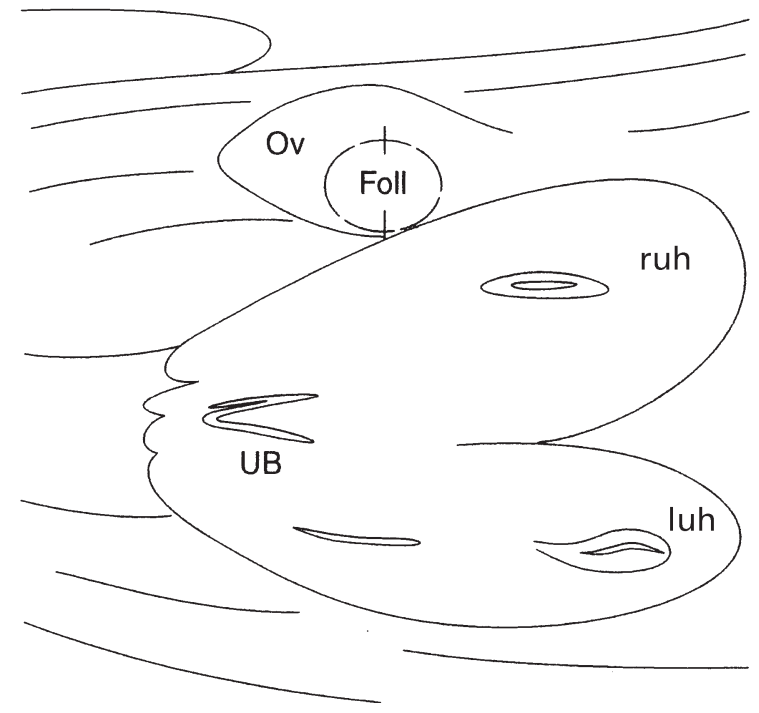

Fig. 5. (a) Coronal-dorsal sonogram showing a longitudinal section of the uterus and right ovary (between the arrows) in a bottlenose dolphin (Tursiops truncatus aduncas). The ovary contains a $13 \mathrm{~mm}$ follicle (indicated between calipers (white crosses)). The image is oriented with the right side uppermost and the cranial aspect to the right. (b) Line diagram of (a). cer: true cervix; foll: follicle; LUH: left uterine horn; Ov: right ovary; RUH: right uterine horn; STR: spermothecal recess; UB: uterine body. Scale bar represents $1 \mathrm{~cm}$.

dolphin was re-stationed after a short interval. If the dolphin did not co-operate, the ultrasonographer and trainer withdrew for a short period before trying again and this approach was usually successful. If the dolphin would not co-operate after several attempts, the procedure was attempted on another day. Keeping the animal in the water

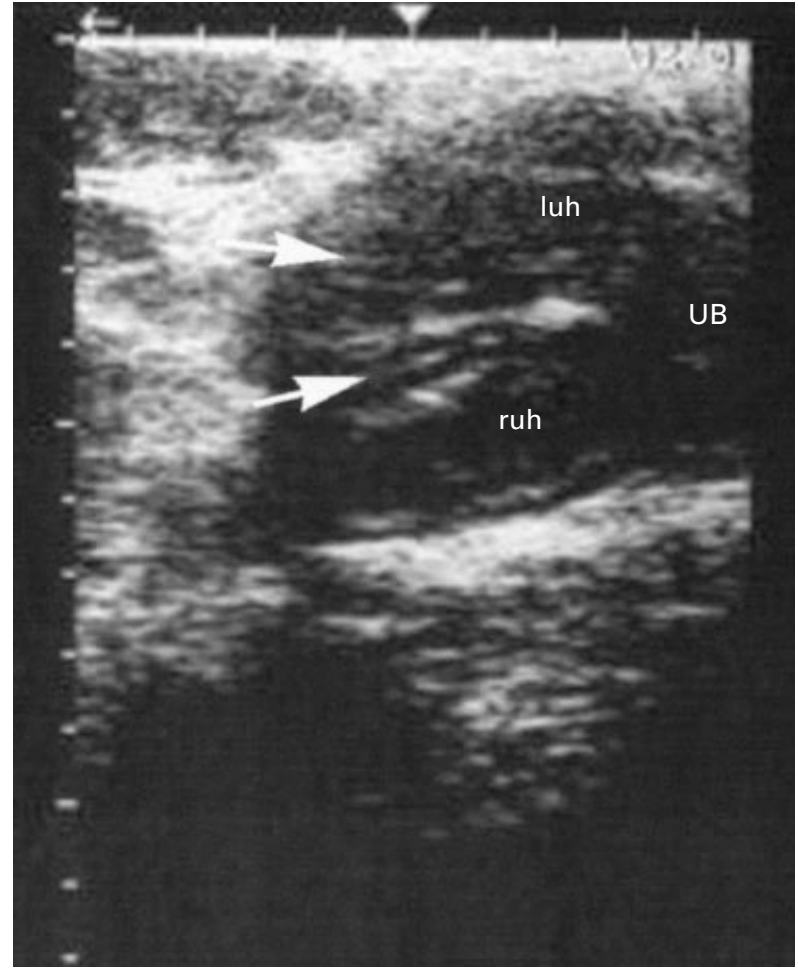

Fig. 6. Coronal-dorsal sonogram showing a longitudinal section of the uterine body and caudal aspect of both uterine horns in a bottlenose dolphin (Tursiops truncatus aduncas). The image is oriented with the left side uppermost and the caudal aspect to the right. Sections of the endometrium are indicated by arrows. luh: left uterine horn; UB: uterine body; ruh: right uterine horn. Scale shown on the image is in $1.0 \mathrm{~cm}$ increments.

is important for several reasons: the area of interest is easily accessible; voluntary co-operation reduces possible stress factors; and examinations can be repeated regularly. This type of behaviour is now a standard part of training in many facilities around the world.

The ultrasonographic appearance of the reproductive organs correlated well with the gross anatomy (Robeck et al., 1994) and was similar in all animals, although the ovarian parenchyma in old females tended to be more echogenic than that in the young females. The increased echogenicity made it more difficult to distinguish the ovaries of old females from surrounding bowel owing to fat or age-related changes, or to an increased number of cortical scars (corpora albicantia) from previous ovulations in the ovaries of old females (Harrison, 1969; Harrison et al., 1972; Perrin et al., 1976; Perrin and Reilly, 1984). Ultrasonographic examination of excised ovaries from a mature female false killer whale (Pseudorca crassidens) revealed multiple, rounded, echogenic foci within the ovarian cortex (F. M. Brook, unpublished). The superficial foci corresponded to corpora albicantia visible on the surface of the ovary. Increased echogenicity, or discrete echogenic foci seen in dolphin ovaries may indicate that 


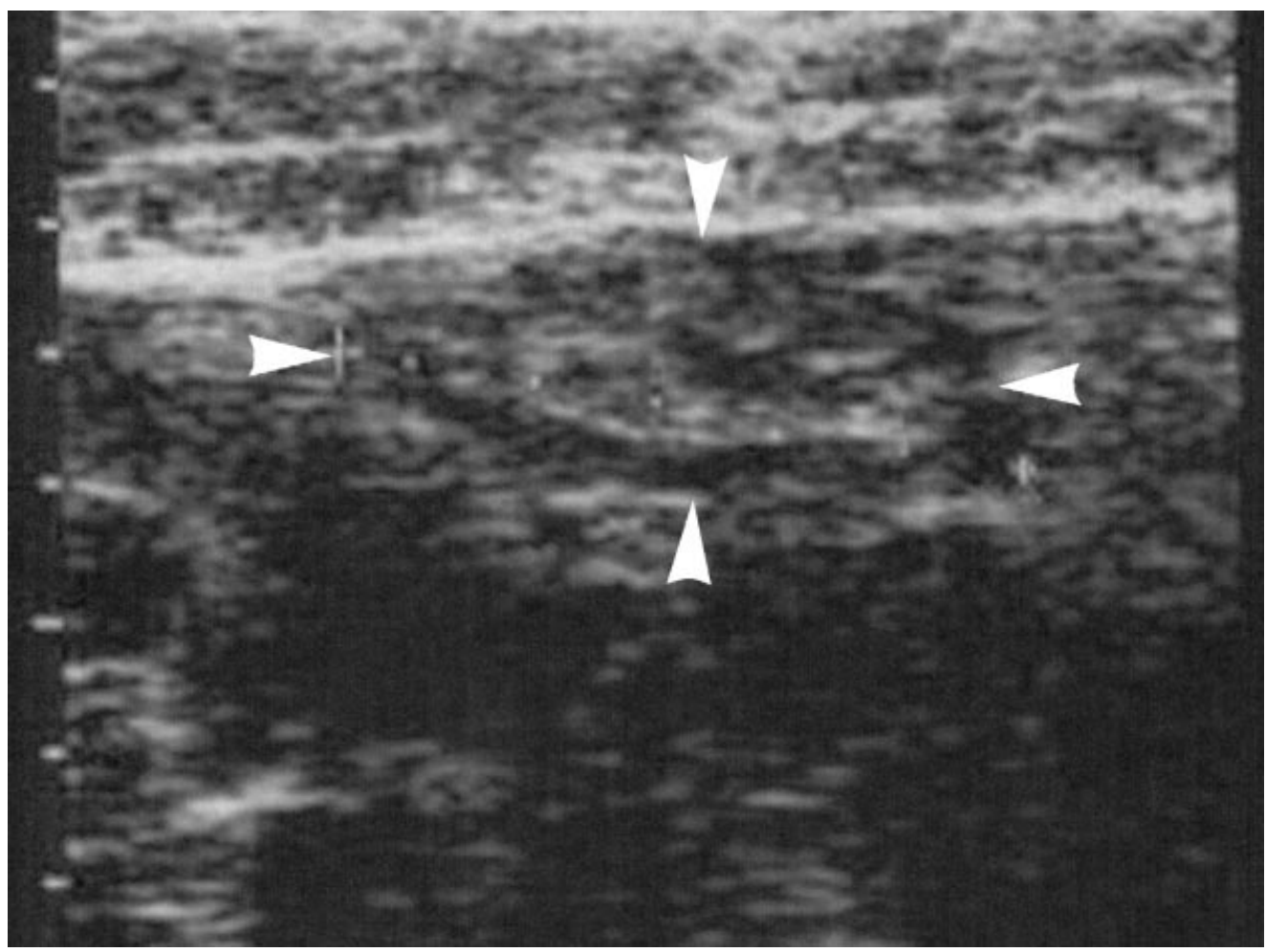

Fig. 7. Longitudinal sonogram through the ovary of an old (> 25 years of age) bottlenose dolphin (Tursiops truncatus aduncas). The ovary (indicated between the arrowheads) is $5.6 \mathrm{~cm}$ in length. The image is oriented with the left side uppermost and the caudal aspect to the right. Scale shown on the lefthand side of the figure is in $1.0 \mathrm{~cm}$ increments.

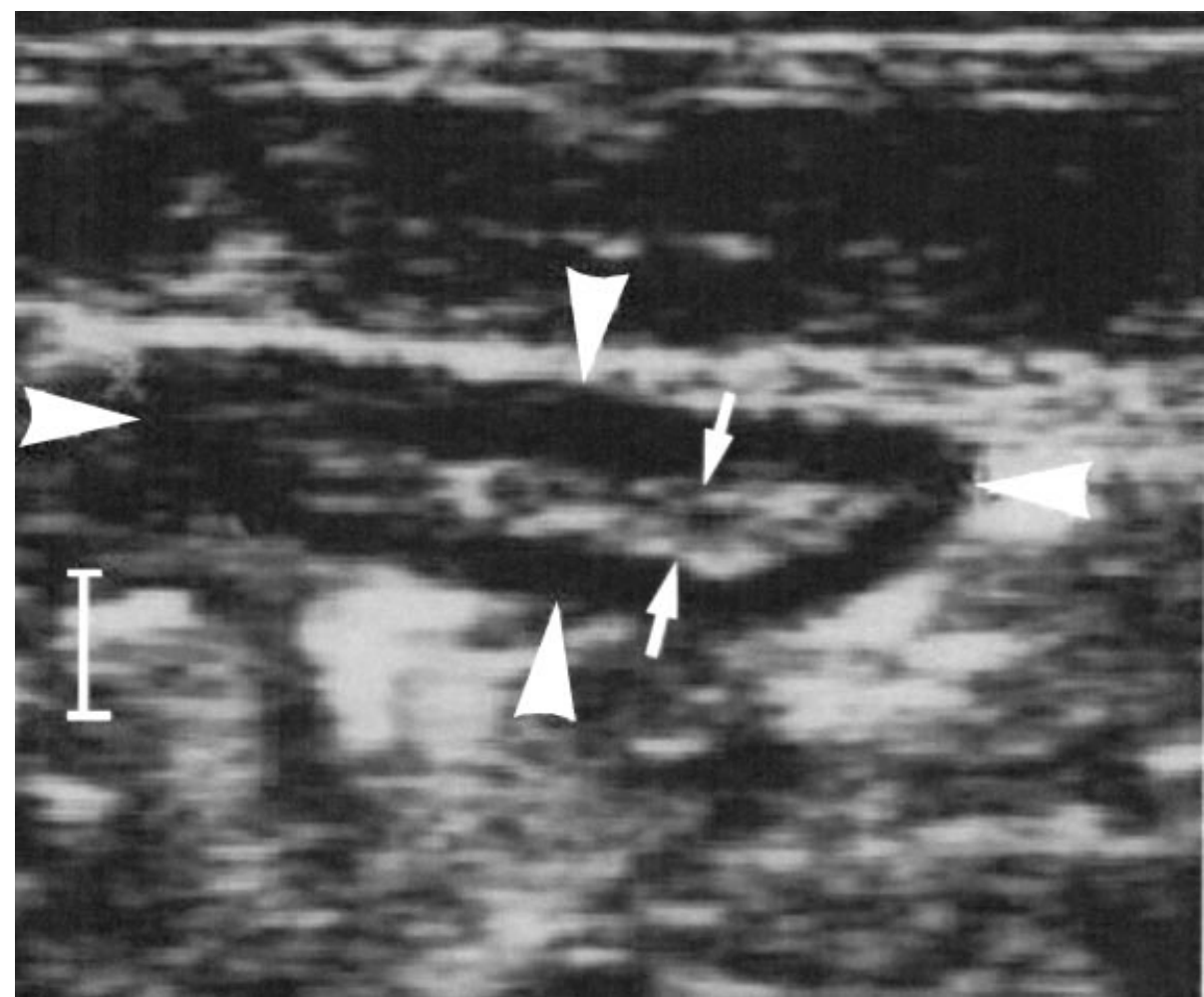

Fig. 8. Longitudinal sonogram through the ovary (indicated between the large arrowheads) of a 6year-old female bottlenose dolphin (Tursiops truncatus aduncas). The image is oriented with the left side uppermost and the caudal aspect to the right. The double, echogenic 'tram line' appearance of the insertion of the mesovarium (small arrows) is obvious. Scale bar represents $1.0 \mathrm{~cm}$. 


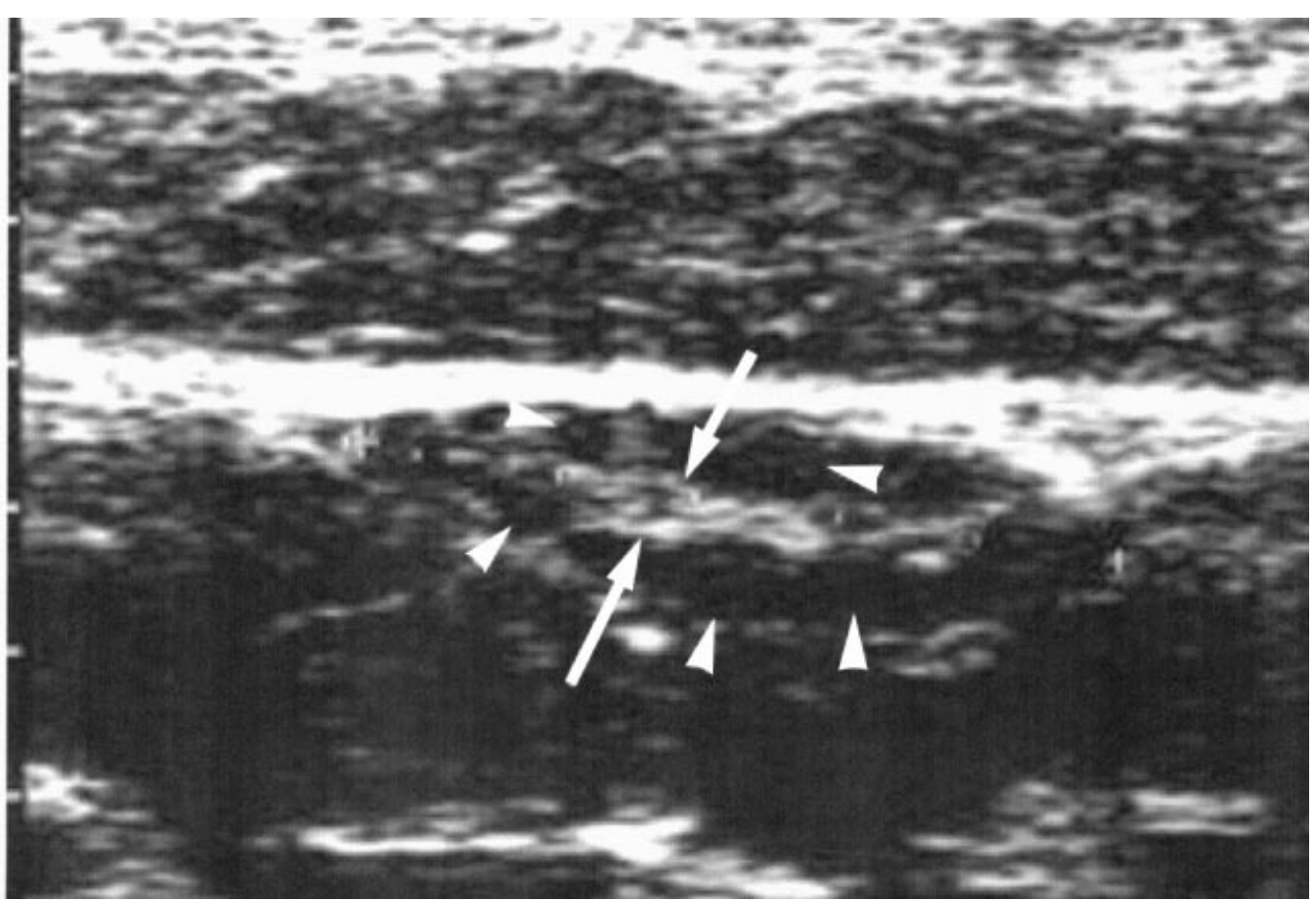

Fig. 9. Longitudinal sonogram through the ovary (extent is indicated by calipers (white crosses), length is $5.4 \mathrm{~cm}$ ) of the same female bottlenose dolphin (Tursiops truncatus aduncas) as shown in Fig. 8 at $>9$ years of age, after five ovulations and one pregnancy. Note the change in shape and echogenicity. Small, quiescent follicles (arrowheads) can be seen throughout the ovarian cortex. Arrows indicate the 'tram line' of the hilum. Images are oriented with the left side uppermost and the caudal aspect to the right. Scale shown on the left-hand side of the figure is in $1.0 \mathrm{~cm}$ increments.

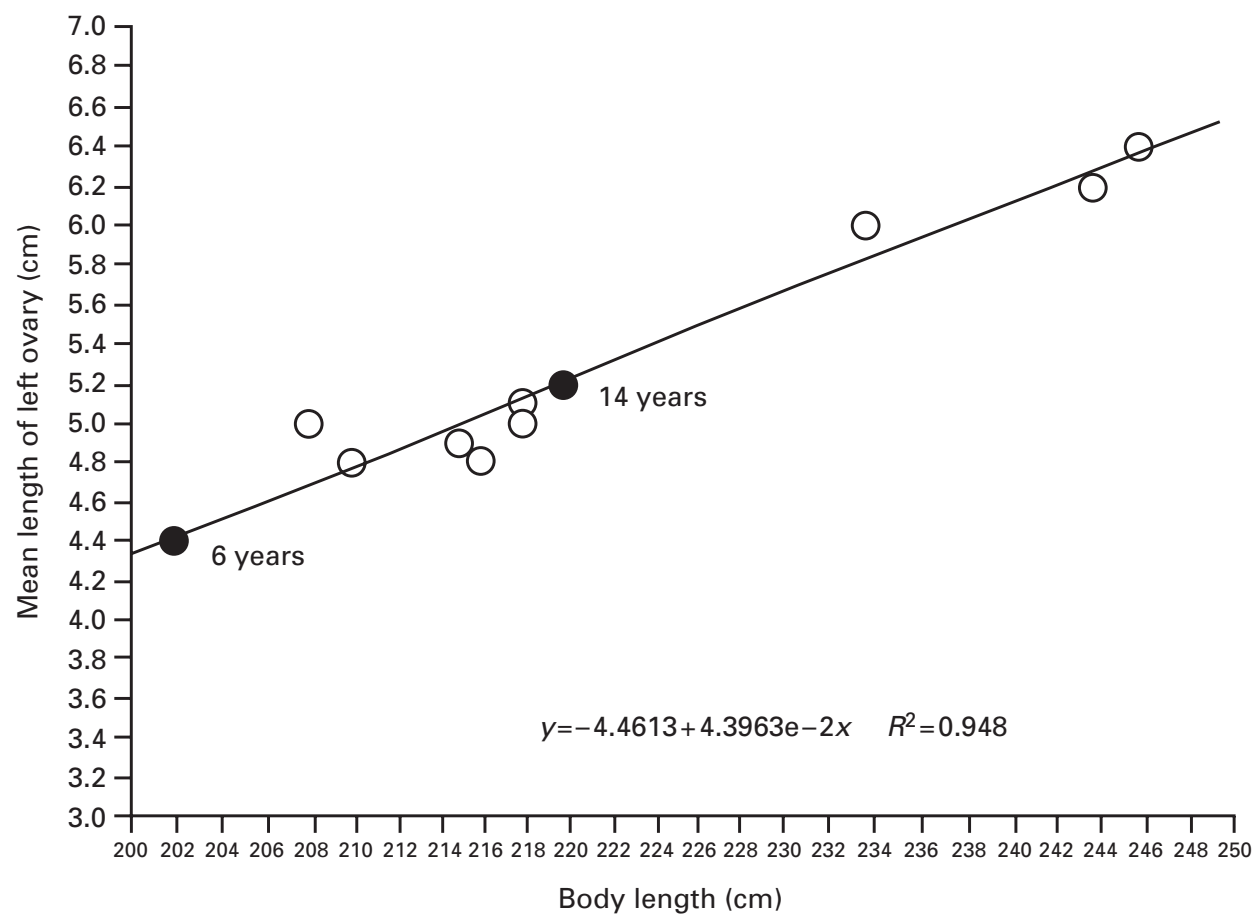

Fig. 10. Scattergram showing comparison between mean length of the left ovary and body length in ten bottlenose dolphins (Tursiops truncatus aduncas). $\mathbf{0}$ : ovarian length and body length in one dolphin at 6 and 14 years of age. 

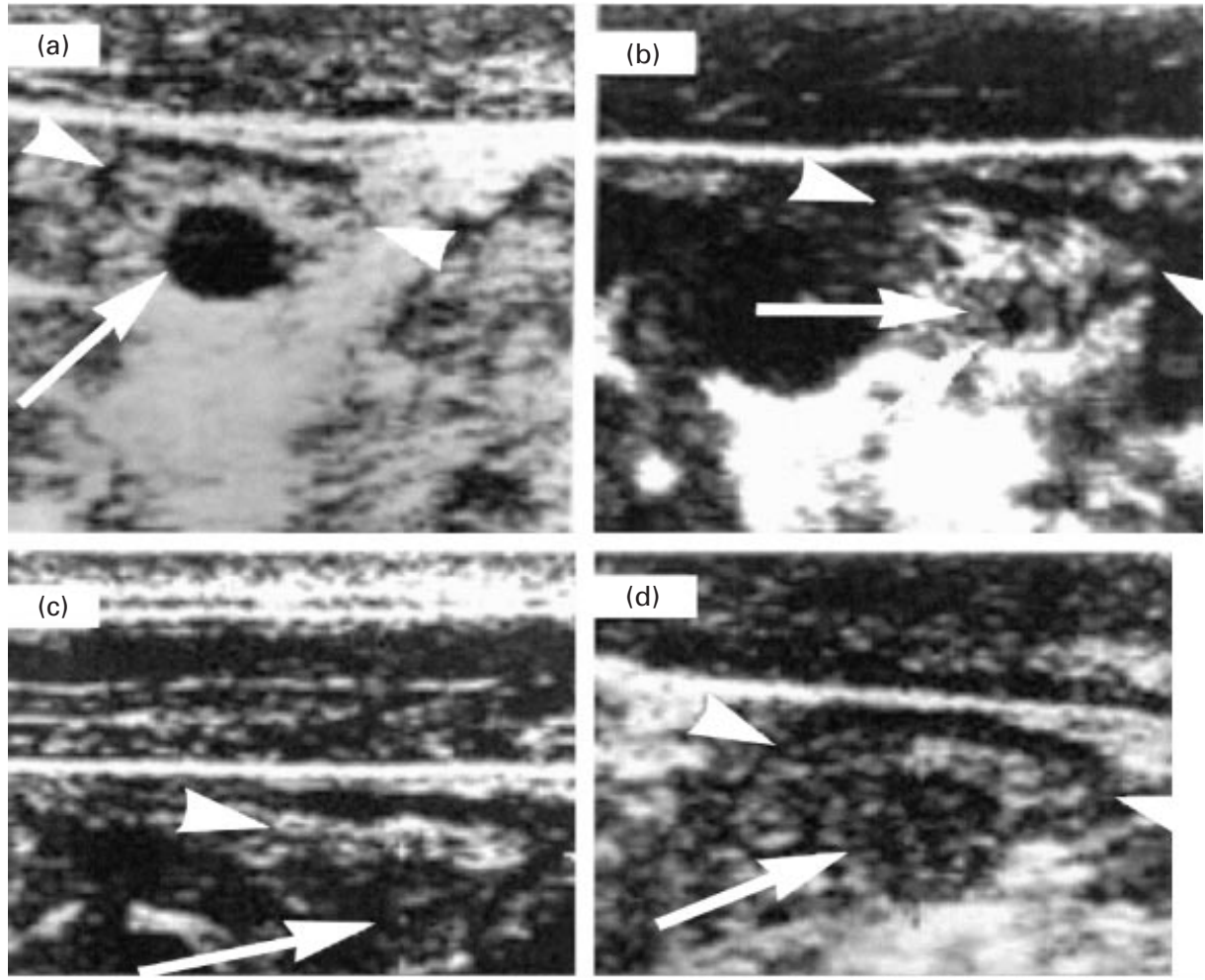

Fig. 11. Images of a particular bottlenose dolphin (Tursiops truncatus aduncas) ovary (indicated between arrowheads) during one cycle. Arrows indicate Graafian follicle (a), site of ruptured follicle immediately after ovulation (b) and corpus luteum 6 days (c) and 10 days (d) after ovulation. Markers between calipers represent $1 \mathrm{~cm}$.

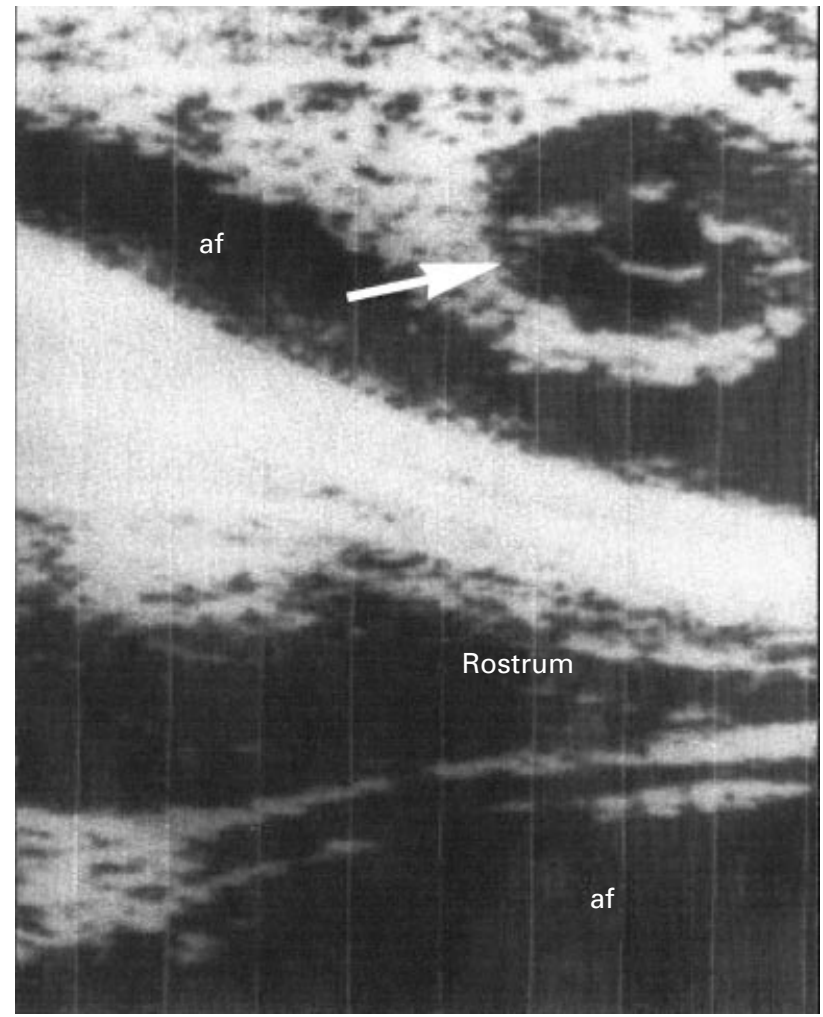

multiple ovulations have occurred and that the female is reproductively mature.

Ultrasonography provides a reliable and repeatable method for examining ovaries in dolphins. With practice, serial examination of the ovaries is relatively easy and animals generally co-operate well in poolside scanning when training is consistent. These techniques also work with other dolphin species, including the Pacific whitesided dolphin (Lagenorhyncus obliquidens), the IndoPacific humpbacked dolphin (Sousa chinensis), the beluga (Delphinapterus leucas) and the killer whale (Orcinus orca) (F. M. Brook, unpublished). Ultrasonographic identification of reproductive events in the present population of dolphins has provided an invaluable tool for controlled breeding and husbandry management.

The support and assistance of the staff of the Zoological Operations and Education Department, Ocean Park Corporation, Hong Kong are gratefully acknowledged.

Fig. 12. A cavitated corpus luteum (arrow) during late pregnancy (week 38 of gestation) in a bottlenose dolphin (Tursiops truncatus aduncas). The corpus luteum measured $3.8 \mathrm{~cm} \times 2.4 \mathrm{~cm}$. The fetal rostrum can be seen ventral to the ovary and corpus luteum. af: amniotic fluid. 


\section{References}

Adams GP, Plotka ED, Asa CS and Ginther OJ (1991) Feasibility of characterizing reproductive events in large, non-domestic species by transrectal ultrasonic imaging Zoo Biology 10 247-253

DeCherney AH, Romero $\mathbf{R}$ and Polan ML (1982) Ultrasound in reproductive endocrinology Fertility and Sterility 37 323-333

Harrison RJ (1969) Reproduction and reproductive organs. In The Biology of Marine Mammals pp 253-348 Ed. HT Andersen. Academic Press, New York

Harrison RJ, Brownell RL and Boice RC (1972) Reproduction and gonadal appearances in some odontocetes. In Functional Anatomy of Marine Mammals pp 361-429 Ed. RJ Harrison. Academic Press, New York

Perrin WF, Coe JM and Zweifel JR (1976) Growth and reproduction of the spotted porpoise, Stenella attenuata, in the offshore eastern tropical Pacific Fisheries Bulletin US 74 229-269

Perrin WF and Reilly SB (1984) Reproductive parameters of dolphins and small whales of the family Delphinidae. In Reproduction in Whales, Dolphins and Porpoises pp 97-125 Eds WF Perrin, RL Brownell and DP McMaster. Report of the International Whaling Commission, Special Issue 6

Robeck TR, Curry BE, McBain JF and Kraemer DC (1994) Reproductive biology of the bottlenose dolphin (Tursiops truncatus) and the potential application of advanced reproductive technologies Journal of Zoo and Wildlife Medicine 25 321-336
Robeck TR, Mathey S, McBain JF and Kraemer DC (1995) Preliminary results of ultrasonographic monitoring of bottlenosed dolphin, Tursiops truncatus, ovarian and uterine responses to exogenous gonadotropins IAAAM Proceedings Vol. 26 pp 103-104 (Abstract)

Stone LR, Phillips B and Sweeney JC (1984) Diagnostic ultrasound of the bottlenose dolphin Annual Proceedings of the American Association of Zoo Vets p 94 (Abstract)

Stone LR (1990) Diagnostic ultrasound in marine mammals. In CRC Handbook of Marine Mammal Medicine: Health, Disease and Rehabilitation pp 235-264 Ed. L Dierauf. CRC Press Inc., Boca Raton, FL

Stone LR, Johnson RL, Sweeney JC and Lewis ML (1999) Fetal ultrasonography in dolphins with emphasis on gestational aging. In Zoo and Wild Animal Medicine. Current Therapy 4 4th Edn pp 501-506 Eds ME Fowler and RE Miller. WB Saunders Company, Philadelphia

Taverne MAM (1991) Applications of two-dimensional ultrasound in animal reproduction Wien Tierarztl Mschr 78 341-345

Williamson P, Gales NJ and Lister S (1990) Use of real-time B-mode ultrasound for pregnancy diagnosis and measurement of fetal growth rate in captive bottlenose dolphins (Tursiops truncatus) Journal of Reproduction and Fertility 88 543-548

Revised manuscript received 25 August 2000.

Accepted 2 October 2000. 